Aus dem Institut/der Klinik für Gynäkologie der Medizinischen Fakultät Charité - Universitätsmedizin Berlin

\title{
DISSERTATION
}

Laparoscopic lymph node dissection should be performed before fertility preserving treatment of patients with cervical cancer

zur Erlangung des akademischen Grades

Doctor medicinae (Dr. med.)

\author{
vorgelegt der Medizinischen Fakultät \\ Charité - Universitätsmedizin Berlin
}

von

Giuseppe Filiberto Vercellino

aus Turin (Italien)

Datum der Promotion: 6. 10. 2014 
1. Abstrakt in Deutsch 3

2. Abstrakt in Englisch $\quad 5$

$\begin{array}{ll}\text { 3. Anteilerklärung } & 7\end{array}$

4. Eidesstattliche Versicherung 8

5. Auszug aus der Journal Summary List (ISI Web of knowledge) 9

$\begin{array}{ll}\text { 6. Publikation } & 12\end{array}$

$\begin{array}{ll}\text { 7. Lebenslauf } & 17\end{array}$

8. Publikationsliste 18

9. Danksagung 24 
Eine Kombination aus neoadjuvanter Chemotherapie und radikaler Trachelektomie erlaubt es auch Frauen mit größeren Zervixkarzinomen (Tumordurchmesser $>2 \mathrm{~cm}$ ) bei Metastasenfreiheit fertilitätserhaltend zu operieren

\begin{abstract}
Abstrakt
Die aktuellen Leitlinien empfehlen die Durchführung einer fertilitätserhaltenden Therapie bei Zervixkarzinom durch radikale Trachelektomie (bei Metastasenfreiheit) nur bis zu einem Tumordurchmesser von $2 \mathrm{~cm}$.

Das Ziel dieser Studie war es die Prognose von Frauen zu untersuchen, die eine fertilitätserhaltende Therapie bei Vorliegen eines größeren Zervixkarzinom (Stadium IB, Tumor $>2 \mathrm{~cm}$ ) wünschten. Dieses Protokoll sah bei Metastasenfreiheit zunächst die Durchführung einer neoadjuvanten Chemotherapie (zur Reduktion des Tumorvolumens) gefolgt von einer radikalen Trachelektomie vor.
\end{abstract}

Methode: Bei allen Studienpatientinnen erfolgte zunächst ein Lymphknotenstaging durch komplette pelvine und paraaortale Lymphonodektomie. Bei Nachweis von einer oder mehrerer Lymphknotenmetastasen wurde die Durchführung einer kombinierten Radiochemotherapie indiziert. Bei Lymphknotenfreiheit erhielten die Patientinnen eine neoadjuvante Chemotherapie und im Anschluss eine radikale Trachelektomie.

Ergebnisse: 18 Frauen wurden in die Studie eingeschlossen. Bei 12 Patientinnen (67\%) wurde im Rahmen des initialen pelvinen und paraaortalen Lymphknotenstagings das Vorliegen von einer oder mehrerer Lymphknotenmetastasen nachgewiesen und daher die Durchführung einer primären 
Radiochemotherapie indiziert. Bei den verbleibenden 6 Frauen (initialer Tumordurchmesser im Mittel ???) wurde keine Metastase nachgewiesen und bei diesen Frauen daher eine neoadjuvante Chemotherapie, gefolgt von einer radikalen Trachelektomie, durchgeführt. Die neoadjuvante Chemotherapie führte bei 3 Frauen zu einer makroskopisch kompletten Tumorrückbildung und bei den verbleibenden 3 Frauen dieser Gruppe zu einer 50\%igen Reduktion des Tumorvolumens. In der Nachbeobachtungszeit von bislang 30 Monaten blieben alle 6 Frauen der Trachelektomiegruppe rezidivfrei, während bei den Frauen mit nachgewiesenen Lymphknotenmetastasen (und nach Durchführung einer kombinierten Radiochemotherapie) in 25\% der betroffenen Frauen (3/12 Frauen) ein Rezidiv auftrat.

Schlussfolgerung: Die initiale Feststellung des Lymphknotenstatus erlaubt es das individuelle Rezidivrisiko genauer zu bestimmen und lässt es vertretbar erscheinen auch Frauen mit größeren Zervixkarzinomen $(>2 \mathrm{~cm}$ ) bei Kinderwunsch durch eine Kombination aus neoadjuvanter Chemotherapie und radikaler Trachelektomie fertilitätserhaltend zu operieren. 


\section{Laparoscopic lymph node dissection should be performed before fertility preserving treatment of patients with cervical cancer.}

\section{Abstract}

Objective: The aim of this study is to assess our results of treatment of women with stage I cervical cancer $>2 \mathrm{~cm}$ in diameter seeking fertility preservation. Treatment consisted of Laparoscopic Pelvic and Paraaortic Lymphadenectomy (LPPLND), and when no nodal metastasis was detected, neoadjuvant chemotherapy (NACT) followed by radical vaginal trachelectomy (RVT). Patients with positive lymph nodes underwent primary chemoradiation. Furthermore, we aim to summarize the literature on this topic.

Methods: A cohort of women younger than 40 years of age with stage I disease $>2$ cm who underwent LPPLND and either NACT and RVT or chemoradiation. Oncological outcome was evaluated prospectively. In addition, a Pubmed search was performed and current literature is summarized.

Results: eighteen women were eligible for this study. Twelve $(67 \%)$ women were diagnosed with metastasis in one or more pelvic and/or paraaortic lymph nodes, and thus received primary chemoradiation. After a mean follow-up of 25.5 months, three out of these 12 women (25\%) developed recurrence. Six women (33\%) underwent NACT and RVT. Three patients experienced complete response to NACT and three patients showed more than $50 \%$ tumor size reduction. After a mean follow-up of 30.6 months all six women are free of recurrence. One patient delivered a healthy infant. 
Eight studies were included in the literature review and used NACT and uterus preservation, however lymph node staging was performed after NACT.

Conclusions: Staging LPPLND allows separating patients in high or low recurrence risk groups. NACT and RVT seems to be safe for women with completely staged stage I cervical cancer $>2 \mathrm{~cm}$ in diameter, whereas even after primary chemoradiation, patients with positive lymph nodes experienced recurrence. Therefore, selection of patients with stage I cervical carcinoma $>2 \mathrm{~cm}$, eligible for fertility preservation should include histopathologic evaluation of lymph node status before any further treatment. 


\section{Eidesstattliche Versicherung}

Ich Giuseppe Filiberto Vercellino versichere an Eides statt durch meine eigenhändige Unterschrift, dass ich die vorgelegte Dissertation mit dem Thema: „Laparoscopic lymph node dissection should be performed before fertility preserving treatment of patients with cervical cancer" selbstständig und ohne nicht offengelegte Hilfe Dritter verfasst und keine anderen als die angegebenen Quellen und Hilfsmittel genutzt habe.

Alle Stellen, die wörtlich oder dem Sinne nach auf Publikationen oder Vorträgen anderer Autoren beruhen, sind als solche in korrekter Zitierung (siehe „Uniform Requirements for Manuscripts (URM)" des ICMJE -www.icmje.org) kenntlich gemacht. Die Abschnitte zu Methodik (insbesondere praktische Arbeiten, Laborbestimmungen, statistische Aufarbeitung) und Resultaten (insbesondere Abbildungen, Graphiken und Tabellen) entsprechen den URM (s.o) und werden von mir verantwortet.

Mein Anteil an der ausgewählten Publikation entspricht dem, der in der untenstehenden gemeinsamen Erklärung mit dem/der Betreuer/in, angegeben ist.

Die Bedeutung dieser eidesstattlichen Versicherung und die strafrechtlichen Folgen einer unwahren eidesstattlichen Versicherung $(\$ 156,161$ des Strafgesetzbuches) sind mir bekannt und bewusst."

Datum

Unterschrift

15.XI:2013 
Ausführliche Anteilserklärung an der erfolgten Publikation

Publikation: Vercellino GF, Piek JM, Schneider A, Köhler C, Mangler M, Speiser D, Chiantera V. Laparoscopic lymph node dissection should be performed before fertility preserving treatment of patients with cervical cancer. Gynecol Oncol. 2012 Sep;126(3):325-9.

Beitrag im Einzelnen (bitte ausführlich ausführen):

Idee: Schneider A, Piek JM, Vercellino GF.

Operationen: Schneider A, Köhler C, Chiantera V(als erste Operateur)

Datensuche: Vercellino GF.

Text: (Einführung, Materialen und Methoden, Resultaten, Tabellen: Vercellino GF, Piek JM)

Text: (Abstrakt, Graphik 1, statistische Aufarbeitung, Diskussion: Vercellino GF).

Datenanalyse: Vercellino GF, Piek JM.

Nachsorge: Vercellino G.F.

Literatur Recherche: Vercellino G.F., Piek JM.

Patientinnen Betreung: Vercellino GF, Piek JM, Schneider A, Köhler C, Mangler M, Speiser D.

Unterschrift, Datum und Stempel des betreuenden Hochschullehrers/der betreuenden Hochschullehrerin

Unterschrift des Doktoranden/der Doktorandin 


\section{ISI Web of Knowledge ${ }^{\text {sm }}$}

\section{Journal Citation Reports ${ }^{\circledast}$}

\section{Journal Summary List}

Journals from:

subject categories OBSTETRICS \& GYNECOLOGY

Sorted by:

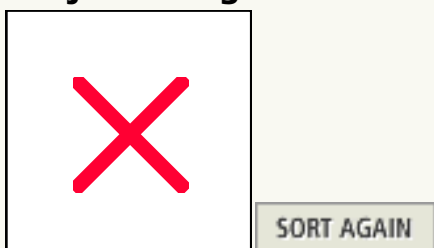

Journals 1 - 20 (of 78)

$\mid\langle\langle\langle\langle[1|\underline{2}| \underline{3} \mid \underline{4}]\rangle\rangle\rangle$

Page 1 of 4

Ranking is based on your journal and sort selections.

\begin{tabular}{|c|c|c|c|c|c|c|c|c|c|c|c|}
\hline \multirow[b]{2}{*}{$\begin{array}{c}\mathrm{Ma} \\
\mathrm{rk}\end{array}$} & \multirow[b]{2}{*}{$\begin{array}{l}\text { Ra } \\
\text { nk }\end{array}$} & \multirow[b]{2}{*}{$\begin{array}{l}\text { Abbreviated } \\
\text { Journal Title } \\
\text { (linked to } \\
\text { journal } \\
\text { information) }\end{array}$} & \multirow[b]{2}{*}{$\begin{array}{l}\text { ISS } \\
\mathrm{N}\end{array}$} & \multicolumn{6}{|c|}{ JCR Data } & \multicolumn{2}{|c|}{$\begin{array}{l}\text { Eigenfactor }^{\circledR} \\
\text { Metrics }\end{array}$} \\
\hline & & & & $\begin{array}{l}\text { Tot } \\
\text { al } \\
\text { Cite } \\
\text { s }\end{array}$ & $\begin{array}{c}\text { Imp } \\
\text { act } \\
\text { Fact } \\
\text { or }\end{array}$ & \begin{tabular}{|c|}
$5-$ \\
Year \\
Imp \\
act \\
Fact \\
or
\end{tabular} & $\begin{array}{l}\text { Immed } \\
\text { iacy } \\
\text { Index }\end{array}$ & $\begin{array}{c}\text { Artic } \\
\text { les }\end{array}$ & $\begin{array}{l}\text { Cit } \\
\text { ed } \\
\text { Hal } \\
\text { f- } \\
\text { life }\end{array}$ & $\begin{array}{l}\text { Eigenfa } \\
\text { ctor }^{\circledR} \\
\text { Score }\end{array}$ & $\begin{array}{c}\text { Article } \\
\text { Influence }^{\circledR} \\
\text { Score }\end{array}$ \\
\hline Г & 1 & $\begin{array}{l}\text { HUM REPROD } \\
\underline{\text { UPDATE }}\end{array}$ & \begin{tabular}{|l}
135 \\
$5-$ \\
478 \\
6
\end{tabular} & $\begin{array}{r}568 \\
5\end{array}$ & $\begin{array}{r}8.84 \\
7\end{array}$ & $\begin{array}{r}9.51 \\
2\end{array}$ & 1.884 & 43 & 6.6 & 0.01350 & 3.068 \\
\hline Г & 2 & $\underline{\text { OBSTET }}$ & $\begin{array}{l}002 \\
9- \\
784 \\
4\end{array}$ & $\begin{array}{r}258 \\
08\end{array}$ & $\begin{array}{r}4.79 \\
8\end{array}$ & $\begin{array}{r}4.90 \\
8\end{array}$ & 1.081 & 307 & 8.9 & 0.04885 & 1.710 \\
\hline Г & 3 & HUM REPROD & \begin{tabular}{|l|}
026 \\
$8-$ \\
116 \\
1
\end{tabular} & $\begin{array}{r}267 \\
33\end{array}$ & $\begin{array}{r}4.67 \\
0\end{array}$ & $\begin{array}{r}4.59 \\
6\end{array}$ & 0.825 & 424 & 8.3 & 0.04542 & 1.314 \\
\hline$\Gamma$ & 4 & FERTIL STERIL & $\begin{array}{l}001 \\
5- \\
028 \\
2\end{array}$ & $\begin{array}{r}282 \\
43\end{array}$ & $\begin{array}{r}4.17 \\
4\end{array}$ & $\begin{array}{r}3.96 \\
0\end{array}$ & 0.885 & 469 & 7.0 & 0.05413 & 1.021 \\
\hline$\Gamma$ & 5 & $\begin{array}{l}\text { GYNECOL } \\
\text { ONCOL }\end{array}$ & $\begin{array}{l}009 \\
0- \\
825 \\
8\end{array}$ & $\begin{array}{r}182 \\
43\end{array}$ & $\begin{array}{r}3.92 \\
9\end{array}$ & $\begin{array}{r}3.92 \\
3\end{array}$ & 0.680 & 409 & 6.7 & 0.03798 & 1.094 \\
\hline Г & 6 & $\frac{\text { AM J OBSTET }}{\underline{\text { GYNECOL }}}$ & $\begin{array}{l}000 \\
2- \\
937 \\
8\end{array}$ & $\begin{array}{r}337 \\
59\end{array}$ & $\begin{array}{r}3.87 \\
7\end{array}$ & $\begin{array}{r}3.82 \\
1\end{array}$ & 1.177 & 351 & $\begin{array}{l}>1 \\
0.0\end{array}$ & 0.05134 & 1.231 \\
\hline
\end{tabular}




\begin{tabular}{|c|c|c|c|c|c|c|c|c|c|c|c|}
\hline$\Gamma$ & 7 & $\begin{array}{l}\text { BJOG-INT J } \\
\text { OBSTET GY } \\
\end{array}$ & \begin{tabular}{|l}
147 \\
$0-$ \\
032 \\
8
\end{tabular} & $\begin{array}{r}124 \\
05\end{array}$ & $\begin{array}{r}3.76 \\
0\end{array}$ & $\begin{array}{r}3.75 \\
4\end{array}$ & 1.315 & 203 & 8.4 & 0.02302 & 1.233 \\
\hline$\Gamma$ & 8 & $\frac{\text { ULTRASOUND }}{\underline{\text { OBST GYN }}}$ & $\begin{array}{l}096 \\
0- \\
769 \\
2\end{array}$ & $\begin{array}{r}849 \\
0\end{array}$ & $\begin{array}{r}3.55 \\
7\end{array}$ & $\begin{array}{r}3.70 \\
8\end{array}$ & 0.756 & 201 & 6.3 & 0.01745 & 0.944 \\
\hline$\Gamma$ & 9 & $\begin{array}{l}\text { SEMIN REPROD } \\
\underline{\text { MED }}\end{array}$ & $\begin{array}{l}152 \\
6- \\
800 \\
4\end{array}$ & $\begin{array}{r}155 \\
0\end{array}$ & $\begin{array}{r}3.21 \\
1\end{array}$ & $\begin{array}{r}3.86 \\
4\end{array}$ & 0.407 & 54 & 4.8 & 0.00551 & 1.223 \\
\hline$\Gamma$ & 10 & MENOPAUSE & $\begin{array}{l}107 \\
2- \\
371 \\
4\end{array}$ & $\begin{array}{r}374 \\
9\end{array}$ & $\begin{array}{r}3.16 \\
3\end{array}$ & $\begin{array}{r}3.13 \\
1\end{array}$ & 1.006 & 167 & 4.5 & 0.01051 & 0.810 \\
\hline$\Gamma$ & 11 & PLACENTA & $\begin{array}{l}014 \\
3- \\
400 \\
4\end{array}$ & $\begin{array}{r}618 \\
7\end{array}$ & $\begin{array}{r}3.11 \\
7\end{array}$ & $\begin{array}{r}3.17 \\
9\end{array}$ & 0.551 & 178 & 6.3 & 0.01293 & 0.834 \\
\hline$\Gamma$ & 12 & $\frac{\text { CONTRACEPTIO }}{\underline{N}}$ & $\begin{array}{l}001 \\
0- \\
782 \\
4\end{array}$ & $\begin{array}{r}498 \\
7\end{array}$ & $\begin{array}{r}3.09 \\
0\end{array}$ & $\begin{array}{r}2.83 \\
7\end{array}$ & 0.518 & 191 & 6.7 & 0.01062 & 0.800 \\
\hline$\Gamma$ & 13 & $\begin{array}{l}\text { BIRTH-ISS } \\
\text { PERINAT C } \\
\end{array}$ & $\begin{array}{l}073 \\
0- \\
765 \\
9\end{array}$ & $\begin{array}{r}170 \\
6\end{array}$ & $\begin{array}{r}2.92 \\
6\end{array}$ & $\begin{array}{r}3.16 \\
1\end{array}$ & 0.146 & 48 & 8.1 & 0.00285 & 0.877 \\
\hline$\Gamma$ & 14 & MATURITAS & $\begin{array}{l}037 \\
8- \\
512 \\
2\end{array}$ & $\begin{array}{r}418 \\
5\end{array}$ & $\begin{array}{r}2.84 \\
4\end{array}$ & $\begin{array}{r}2.66 \\
7\end{array}$ & 0.491 & 173 & 5.8 & 0.00945 & 0.701 \\
\hline$\Gamma$ & 15 & $\begin{array}{l}\text { SEMIN } \\
\text { PERINATOL }\end{array}$ & $\begin{array}{l}014 \\
6- \\
000 \\
5\end{array}$ & $\begin{array}{r}202 \\
4\end{array}$ & $\begin{array}{r}2.81 \\
4\end{array}$ & $\begin{array}{r}2.93 \\
2\end{array}$ & 0.284 & 67 & 6.7 & 0.00464 & 1.003 \\
\hline$\Gamma$ & 16 & PRENATAL DIAG & \begin{tabular}{|l|}
019 \\
$7-$ \\
385 \\
1
\end{tabular} & $\begin{array}{r}521 \\
8\end{array}$ & $\begin{array}{r}2.68 \\
3\end{array}$ & $\begin{array}{r}2.33 \\
4\end{array}$ & 0.568 & 213 & 6.7 & 0.01149 & 0.666 \\
\hline$\Gamma$ & 17 & $\frac{\text { REPROD }}{\text { BIOMED ONLINE }}$ & $\begin{array}{l}147 \\
2- \\
648 \\
3\end{array}$ & $\begin{array}{r}477 \\
6\end{array}$ & $\begin{array}{r}2.67 \\
5\end{array}$ & $\begin{array}{r}2.52 \\
3\end{array}$ & 0.718 & 174 & 4.8 & 0.01405 & 0.699 \\
\hline$\Gamma$ & 18 & $\frac{\text { CURR OPIN }}{\text { OBSTET GYN }}$ & $\begin{array}{l}104 \\
0- \\
872 \\
X\end{array}$ & $\begin{array}{r}180 \\
9\end{array}$ & $\begin{array}{r}2.63 \\
7\end{array}$ & $\begin{array}{r}2.50 \\
8\end{array}$ & 0.348 & 66 & 5.5 & 0.00574 & 0.839 \\
\hline$\Gamma$ & 19 & $\begin{array}{l}\text { CLIN } \\
\text { PERINATOL }\end{array}$ & $\begin{array}{l}009 \\
5- \\
510 \\
8\end{array}$ & $\begin{array}{r}156 \\
6\end{array}$ & $\begin{array}{r}2.58 \\
2\end{array}$ & $\begin{array}{r}2.62 \\
9\end{array}$ & 0.233 & 60 & 6.9 & 0.00390 & 0.913 \\
\hline$\Gamma$ & 20 & $\frac{\text { OBSTET }}{\text { GYNECOL SURV }}$ & $\begin{array}{l}002 \\
9-\end{array}$ & $\begin{array}{r}195 \\
5\end{array}$ & $\begin{array}{r}2.51 \\
4\end{array}$ & $\begin{array}{r}2.98 \\
9\end{array}$ & 0.167 & 36 & $\begin{array}{l}>1 \\
0.0\end{array}$ & 0.00298 & 0.948 \\
\hline
\end{tabular}




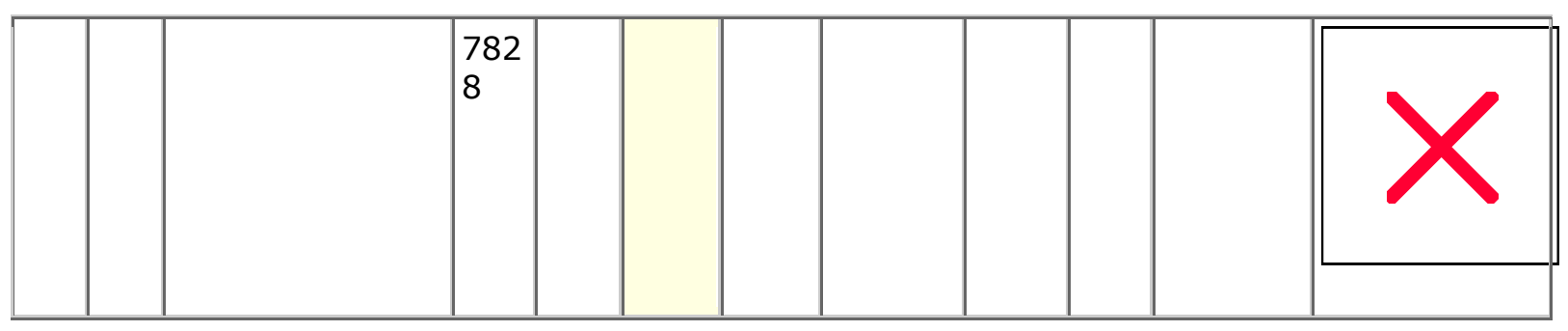

MARK ALL UPDATE MARKED LIST

Journals $1-20$ (of 78) $\quad|\langle\langle\rangle\langle[1|\underline{2}| \underline{3} \mid \underline{4}]\rangle\rangle\rangle \mid \quad$ Page 1 of 4

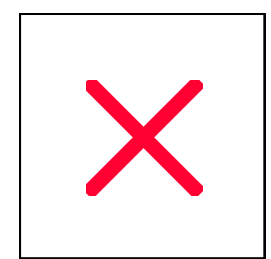


Gynecologic Oncology 126 (2012) 325-329

Corresponding author.

E-mail address: enonloso1@hotmail.com (G.F. Vercellino).

0090-8258/\$ - see front matter @ 2012 Elsevier Inc. All rights reserved.

Gynecologic Oncology

journal homepage: www.elsevier.com/locate/ygyno

Laparoscopic lymph node dissection should be performed before fertility preserving treatment of patients with cervical cancer

Giuseppe F. Vercellino a,, Jurgen M.J. Piek a,b, Achim Schneider a, Christhardt

Köhler a, Mandy Mangler a,

Dorothee Speiser a, Vito Chiantera a

a Charité University, Department of Gynaecology, Campus Benjamin Franklin and Campus Mitte, Hindenburgdamm 30, and Charitéplatz 1, Berlin, Germany

b Comprehensive Cancer Centre South Location TweeSteden Hospital, PO Box 90107, 5000 LA Tilburg, The Netherlands

doi:10.1016/j.ygyno.2012.05.033 


\section{LEBENSLAUF}

"Mein Lebenslauf wird aus datenschutzrechtlichen Gründen in der elektronischen Version meiner Arbeit nicht veröffentlicht." 


\section{Publikationsliste}

1. Vercellino GF, Piek JM, Schneider A, Köhler C, Mangler M, Speiser D, Chiantera V. Laparoscopic lymph node dissection should be performed before fertility preserving treatment of patients with cervical cancer. Gynecol Oncol. 2012 Sep;126(3):325-9. IF: $\mathbf{3 . 9 2 9}$

2. Marnitz S, Köhler C, Affonso RJ, Schneider A, Chiantera V, Tsounoda A, Vercellino F. Validity of laparoscopic staging to avoid adjuvant chemoradiation following radical surgery in patients with early cervical cancer. Oncology. 2012;83(6):346-53. IF: 2.165

3. Vercellino GF, Erdemoglu E, Chiantera V, Vasiljeva K, Drechsler I, Cichon G, Schneider A, Böhmer G. Validity of the colposcopic criteria inner border sign, ridge sign, and rag sign for detection of high-grade cervical intraepithelial neoplasia. Obstet Gynecol. 2013 Mar;121(3):624-31. IF: 4.798

4. Vercellino G, Erdemoglu E, Joe A, Hopfenmueller W, Holthaus B, Köhler C, Schneider A, Hasenbein K, Chiantera V. Laparoscopic temporary clipping of 
uterine artery during laparoscopic myomectomy. Arch Gynecol Obstet. 2012 Nov;286(5):1181-6. IF:1.330

5. G. F. Vercellino, V. Chiantera, J. Gaßmann, E. Erdemoglu, I. Drechsler, S. Frangini, A. Schneider, G. Böhmer. Prospective comparison of loop excision under colposcopic guidance versus vitom guidance Geburtshilfe und Frauenheilkunde 72 (10) , pp. 945-948. IF: $\mathbf{0 . 8 4 8}$

6. Vercellino GF, Erdemoglu E, Kyeyamwa S, Drechsler I, Vasiljeva J, Cichon G, Schneider A. Evaluation of the VITOM in digital high-definition video exocolposcopy. J Low Genit Tract Dis. 2011 Oct;15(4):292-5. IF: 1.207

7. Schneider A, Erdemoglu E, Vercellino G.F. author reply 480-1. . VITOM in digital high-definition video exocolposcopy. J Low Genit Tract Dis. 2012 Oct; 16(4):480. IF: $\mathbf{1 . 2 0 7}$

8. Schneider A, Köhler C, Chiantera V, Vercellino G.F. Cervical cancer and intraepithelial neoplasia. Monographs in Virology 28 , pp. 38-49

\section{Coauthorship:}

9. Taube ET, Frangini S, Caselitz J, Chiantera V, Pahl S, Vercellino GF, Ullrich A, von Cotta G, Dietel M, Younes S, Denkert C. Ligneous cervicitis in a woman with plasminogen deficiency associated with an atypical form of 
microglandular hyperplasia: a case report and review of literature. Int $\mathrm{J}$ Gynecol Pathol. 2013 May;32(3):329-34. IF: 1.413

10. Barcena de Arellano ML, Arnold J, Lang H, Vercellino GF, Chiantera V, Schneider A, Mechsner S. Evidence of neurotrophic events due to peritoneal endometriotic lesions. Cytokine. 2013 May;62(2):253-61. IF: 2.518

11. Fiebitz A, Fritsch M, Reichelt U, Ruester C, Chiantera V, Vercellino GF, Darwish A, Schneider A, Mechsner S. Optimized culture conditions for tissue explants of uterine leiomyoma. Clin Lab. 2012;58(11-12):1153-64. IF: $\mathbf{0 . 9 2 0}$

12. Arnold J, Vercellino GF, Chiantera V, Schneider A, Mechsner S, Barcena de Arellano ML. Neuroimmunomodulatory alterations in non-lesional peritoneum close to peritoneal endometriosis. Neuroimmunomodulation. 2013;20(1):9-18. IF: 1.835

13. Barcena de Arellano ML, Arnold J, Sacher F, Blöchle M, Staube M, Bartley J, Vercellino GF, Chiantera V, Schneider A, Mechsner S. Eutopic endometrium from women with endometriosis does not exhibit neurotrophic properties. J Neuroimmunol. 2012 Aug 15;249(1-2):49-55. IF: $\mathbf{3 . 0 3 3}$

14. Arnold J, Barcena de Arellano ML, Rüster C, Vercellino GF, Chiantera V, Schneider A, Mechsner S. Imbalance between sympathetic and sensory innervation in peritoneal endometriosis. Brain Behav Immun. 2012 Jan;26(1):132-41. IF: $\mathbf{5 . 6 1 2}$ 
15. Barcena de Arellano ML, Arnold J, Vercellino GF, Chiantera V, Ebert AD, Schneider A, Mechsner S. Influence of nerve growth factor in endometriosisassociated symptoms. Reprod Sci. 2011 Dec;18(12):1202-10. I IF: 2.064

16. Chiantera V, Erdemoglu E, Vercellino G, Straube M, Schneider A. Laparoscopic management of external iliac artery injury using yasargil clamps and intracorporeal suture. J Minim Invasive Gynecol. 2011 Jul-Aug;18(4):5169. IF: $\mathbf{1 . 6 0 8}$

17. Marnitz S, Budach V, Weißer F, Burova E, Gebauer B, Vercellino FG, Köhler C. Rectum separation in patients with cervical cancer for treatment planning in primary chemo-radiation. Radiation Oncology 7 (1), art. no. 109 IF: 2.107

18. Barcena de Arellano ML, Arnold J, Vercellino F, Chiantera V, Schneider A, Mechsner S. Overexpression of nerve growth factor in peritoneal fluid from women with endometriosis may promote neurite outgrowth in endometriotic lesions. Fertil Steril. 2011 Mar 1;95(3):1123-6.

19. Kavallaris A, Köhler C, Diebolder H, Vercellino F, Krause N, Schneider A. Repair of prolapse with vaginal sacrocolporectopexy: technique and results. Eur J Obstet Gynecol Reprod Biol. 2005 Oct 1;122(2):237-42. Epub 2005 Jun 9. IF: $\mathbf{1 . 8 4 3}$ 
20. Alba E, Tufano L, De' Sperati C, Vercellino F, Lauricella A, De Grandis T. [New aspects concerning the corpus luteum]. Minerva Ginecol. 1992 Jun;44(6):277-81. [Article in Italian]

\section{ACCEPTED FOR PUBLICATION:}

21.Giuseppe F. Vercellino, Christardt Koehler, MD, Evrim Erdemoglu, Mandy Mangler, Malgorzata Lanowska, Al-Hakeem Malak, Achim Schneider, Vito Chiantera. Laparoscopic pelvic lymphadenectomy in 32 pregnant patients with cervical cancer:

rationale, description of the technique and outcome. International Journal of Gynecological Cancer IF: 1.941

22. Malgorzata Lanowska, Mandy Mangler, Dorothee Speiser, Carolin Bockholdt, Achim Schneider, Christhardt Koehler, Jekaterina Vasiljeva, Al-Hakeem Malak, Giuseppe F Vercellino. Radical vaginal trachelectomy after laparoscopic staging and neoadjuvant chemotherapy preserves fertility in women with early stage cervical cancer over $2 \mathrm{~cm}$ : oncologic, fertility and neonatal outcome in a series of 20 patients. International Journal of Gynecological Cancer IF: 1.941

23. Vito Chiantera, Martina Rossi, Pierandrea De laco, Christardt Koehler, Simone Marnitz, Anna Fagotti, Francesco Fanfani, Fabio Parazzini, Riccardo Schiavina, Giovanni Scambia, Achim Schneider, Giuseppe Filiberto 
Vercellino. Morbidity after Pelvic Exenteration for gynecological malignancies: a retrospective multicentric study of 230 patients. Int J Gynecol Cancer IF:

\subsection{1}

24. Vito Chiantera, Martina Rossi, Pierandrea De laco, Christardt Koehler, Simone Marnitz, Valerio Gallotta, Alessandro Pasquale Margariti, Fabio Parazzini, Giovanni Scambia, Achim Schneider, Giuseppe Filiberto Vercellino. Pelvic exenteration for recurrent endometrial adenocarcinoma: a retrospective multiinstitutional study about 21 patients. International Journal of Gynecological Cancer

IF: 1.941

25. Vito Chiantera, Martina Rossi, Pierandrea De laco, Christardt Koehler, Simone Marnitz, Gabriella Ferrandina, Francesco Legge, Fabio Parazzini, Giovanni Scambia, Achim Schneider, Giuseppe Filiberto Vercellino. Survival after curative pelvic exenteration for primary or recurrent cervical cancer: a retrospective multicentric study of 167 patients. International Journal of Gynecological Cancer IF: $\mathbf{1 . 9 4 1}$ 


\section{Danksagung}

Zu aller erst möchte ich Herr Professor Achim Schneider, Direktor im Ruhestand der Klinik für Gynäkologie an der Charité Campus Benjamin Franklin und Campus Mitte herzlich danken für die Möglichkeit meiner wissenschaftlichen und klinischen Tätigkeiten zu verbessern.

Meine Kolleginnen und Kollegen, die mir sowohl Freund und Vorbilder, als auch wissenschaftliche Begleiter und Koautoren, gilt meine herzlicher Dank: PhD. Jürgen Piek, Prof. Christhardt Köhler, Dr. Vito Chiantera, Dr. Mandy Mangler, Dr. Dorothee Speiser, und Prof. Schneider.

Mein besonderer Dank gilt Frau Dr. Dr. Petra Spek, die mir als ich nach Berlin kam extrem geholfen hat.

Meine Eltern, papá Agostino Vercellino und mamma Simonetta Bottero, danke ich von ganzem Herzen für die Unterstützung als ich Italien verlies und die zahlreiche, liebevolle Ratschläge über die Jahre.

Ich bedanke mich herzlich bei Frau Heike Berger für die Korrekturen der Deutsche Sprache und bei Frau Manuela Hirche für Ihre Hilfe bei der Abgabe der Doktorarbeit.

Meiner wundervollen Frau, Diana Comon Longos gilt mein allergrößter Dank für die Liebe, die Geduld und die kontinuierliche Unterstützung meiner Arbeit. Ohne Sie wäre diese Arbeit nie möglich gewesen. 